\title{
Influences of Material Parameters on Power Spinning Process of Large Ellipsoidal Heads with Variable Thicknesses
}

\author{
Jinhui Zhang ${ }^{1, \mathrm{a}}$, Mei Zhan ${ }^{2, \mathrm{~b}}$, Zhongtai He ${ }^{1, \mathrm{c}}$, Guiling Chen ${ }^{1, \mathrm{~d}}$, Guoan Liang ${ }^{1, \mathrm{e}}$, \\ Guojun Fan ${ }^{1, f}$ \\ 1Jinxi Industries Group Co., Ltd., Taiyuan, Shanxi 030027, PR China \\ ${ }^{2}$ State Key Laboratory of Solidification Processing, School of Materials Science and Engineering, \\ Northwestern Polytechnical University, P.O. Box 542, Xi'an, Shaanxi 710072, PR China \\ azhangjinhui79910@163.com, bzhanmei@nwpu.edu.cn, ${ }^{c} 13994280691 @ 139 . c o m$, \\ dchenguiling@sina.com, ${ }^{\mathrm{e}}$ liangguoan@sina.com, ${ }^{\mathrm{f}}$ 1123058804@qq.com
}

Keywords: power spinning, LEHVT, material parameters, numerical simulation, ABAQUS

Abstract. During the power spinning process of large ellipsoidal heads with variable thicknesses (LEHVT), material parameters of the blank have a significant effect on the quality of LEHVT and the determination of forming parameters. In this paper, the influences of hardening exponent, yield stress and elastic modulus on spinning force, degree of inhomogeneous deformation and unfitability degree are investigated by using 3D-FE numerical simulation under ABAQUS software environment. The results show that, spinning force increases with the increase of hardening exponent, yield stress and elastic modulus. Degree of inhomogeneous deformation increases with the decrease of hardening exponent and the increase of elastic modulus and yield stress. Unfitability degree increases with the decrease of elastic modulus and the increase of hardening exponent and yield stress. The achievements may provide not only an important reference and basis for selecting spinning equipment, but also a guide for quality and process control of the power spinning process of LEHVT.

\section{Introduction}

As well known, power spinning is an advanced flexible technique used to manufacture various head pressure vessels in fields of aeronautics, astronautics, weaponry, and so on[1]. Especially, it is more suitable for forming LEHVT due to unmatched technical and economic advantages such as high precision, high capability, high efficiency, and low cost[2]. During the metal forming process, the force and power parameters and the quality of the workpiece depend on material parameters and forming parameters. Furthermore, material parameters have effect on the determination of forming parameters [3, 4]. Hence, it is important in term of process and quality control to study the influences of material parameters on the power spinning process of LEHVT.

Recently, more and more scholars have placed emphasis on the effects of material parameters on the spinning process. Huang Liang et al.[5] discussed the effects of elastic modulus, hardening exponent and yield stress on splitting force, splitting spinning moment, degree of inhomogeneous deformation and quality of flange in the splitting spinning process. However, head spinning is a complicated plastic forming process under multi-factor effects. Until now, some scholars have studied the process by using analytic method and FE numerical simulation[6], but very few have focused on the study on the influences of material parameters on the process. Especially, compared with the traditional head spinning process and other spinning processes, the power spinning process of LEHVT is quite complex because of large diameter and small thickness of such heads. So, it is very difficult to understand the process purely by analytic or experimental method. Therefore, in this study, in order to control and improve quality of deformed heads, the influences of material parameters including hardening exponent $n$, yield stress $\sigma_{s}$ and elastic modulus $E$ on spinning force, degree of inhomogeneous deformation and unfitability degree are investigated by using the three dimensional (3D) elastic-plastic dynamic FE numerical simulation under the ABAQUS software environment. 


\section{Research method}

Establishment of 3D-FE model. During the power spinning process of LEHVT, the blank is formed into the desired head under the action of the roller, and the thickness of the head gradually decreases along the negative y-axis direction in accordance with the sine law, as shown in Fig. 1. The interior surface generatrix of LEHVT is described as the following equation:

$$
\frac{x^{2}}{a^{2}}+\frac{y^{2}}{b^{2}}=1
$$

Where $a$ is the semi-major axis, $b$ is the semi-minor axis, and $a=2 b$.

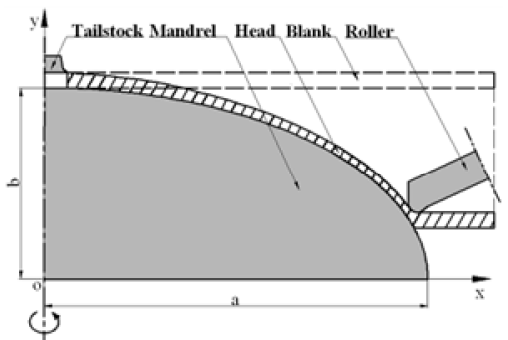

Fig.1 Sketch of power spinning of LEHVT

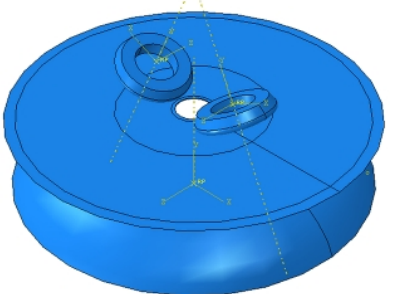

Fig.2 FE model of power spinning of LEHVT

Under the comprehensive consideration of the characteristics of power spinning of LEHVT, a 3D elastic-plastic FE model is developed based on ABAQUS/Explicit, as shown in Fig. 2. To simplify the process, tie constraint between the bottom of the blank and the mandrel is adopted to ensure that the blank can rotate in synchronization with the mandrel. Thus, the tailstock is omitted in the model, which helps to improve the computational efficiency. Moreover, key technologies involved in modeling are dealt with reasonably, including the selection of element types, meshing technology, treatment of boundary conditions and the implementation of variable mandrel rotation velocity and constant roller feed rate, and so on.

Verification of the model. Using the established FE model as mentioned previously, the head spinning process of D406A is simulated. The simulation conditions are listed in Table 1 and Table 2. The material used in the simulation is supposed as an isotropic, homogeneous, elastic-plastic material following the Von-Mises yield criterion. The material constitutive equation of $\sigma=K \varepsilon^{n}$ is adopted, where $\sigma$ is the true stress and $\varepsilon$ is the true strain. The main material parameters used in the simulation are listed in Table 3. The experimental verification has been performed in literature[7].

Table 1 Geometric dimensions and process parameters of model

\begin{tabular}{ll}
\hline Parameters & Values \\
\hline Blank diameter $D_{0}(\mathrm{~mm})$ & 1840 \\
Blank thickness $t_{0}(\mathrm{~mm})$ & 10 \\
Roller diameter $D_{\rho}(\mathrm{mm})$ & 440 \\
Roller nose radius $r_{\rho}(\mathrm{mm})$ & 24 \\
Roller width $H(\mathrm{~mm})$ & 84 \\
Mandrel semi-major axis $a(\mathrm{~mm})$ & 1000 \\
Mandrel semi-minor axis $b(\mathrm{~mm})$ & 500 \\
Roller feed ratio $f(\mathrm{~mm} / \mathrm{r})$ & 1.2 \\
Initial mandrel speed $n_{M}(\mathrm{rpm})$ & 75 \\
\hline
\end{tabular}


Table 2 Deviation ratio

\begin{tabular}{ccccccccc}
\hline$x(\mathrm{~mm})$ & $(300-440]$ & $(440-552]$ & $(552-680]$ & $(680-740]$ & $(740-804]$ & $(804-860]$ & $(860-900]$ & $(900-920]$ \\
\hline $\begin{array}{c}\text { Deviation } \\
\text { ratio }\end{array}$ & -0.03 & -0.035 & -0.038 & -0.04 & -0.045 & -0.05 & -0.06 & -0.08 \\
\hline
\end{tabular}

Table 3 Material parameters

\begin{tabular}{ll}
\hline Parameters & Values \\
\hline $\begin{array}{l}\text { Density } \rho\left(\mathrm{kg} / \mathrm{m}^{3}\right) \\
\text { Young's modulus } E\end{array}$ & 7800 \\
$\begin{array}{l}\mathrm{GPa}) \\
\text { Poisson's ratio } v\end{array}$ & 0.27 \\
Yield stress $\sigma_{s}(\mathrm{Mpa})$ & 507 \\
Strength factor $K$ & 1090 \\
Hardening exponent & 0.18 \\
$n$ & \\
\hline
\end{tabular}

Determination of calculation conditions. Generally, the material properties of the blank are mainly determined by some critical material parameters such as elastic modulus $E$, hardening exponent $n$, and yield stress $\sigma_{s}$. As long as changing any one of them will obtain various materials with different material properties. Therefore, the three parameters are selected as the vital effect factors in this study. The material used in the simulation is D406A, and the simulation conditions are the same as those in Table 1, Table 2 and Table 3. Based on the experimental measurement, elastic modulus $E$, hardening exponent $n$ and yield stress $\sigma_{s}$ are in the range of 189.960 201.805 GPa, 0.15 0.201 and 477 513 $\mathrm{MPa}$, respectively. More widespread material properties of the blank should be reflected through the selection of these parameters. Thus, employing the established FE model, the influences of the material parameters are investigated in this work. The calculation conditions are considered as follows.

Case 1: select $E=167.5,182.5,197.5,212.5,227.5 \mathrm{GPa}$, but keep the other parameters constant as listed in Table 1, Table 2 and Table 3.

Case 2: select $n=0.12,0.15,0.18,0.21,0.24$, but keep the other parameters constant as listed in Table 1, Table 2 and Table 3.

Case 3: select $\sigma_{\mathrm{s}}=457,482,507,532,557 \mathrm{MPa}$, but keep the other parameters constant as listed in Table 1, Table 2 and Table 3.

\section{Result and discussion}

Spinning force. During the power spinning process of LEHVT, the spinning force is a very important parameter for selecting the materials of the blank and determining the equipments in terms of force and power parameters. Fig. 3 shows the influences of the material parameters on the spinning force. It can be seen that when $E, n$ or $\sigma_{s}$ increases, higher spinning force occurs. And the influence of $n$ on the spinning force is more notable. The spinning force is directly related to the equivalent stress in the spinning process. The equivalent stress variations obtained from FE simulation results have been studied to evaluate the spinning force, as shown in Fig.4.
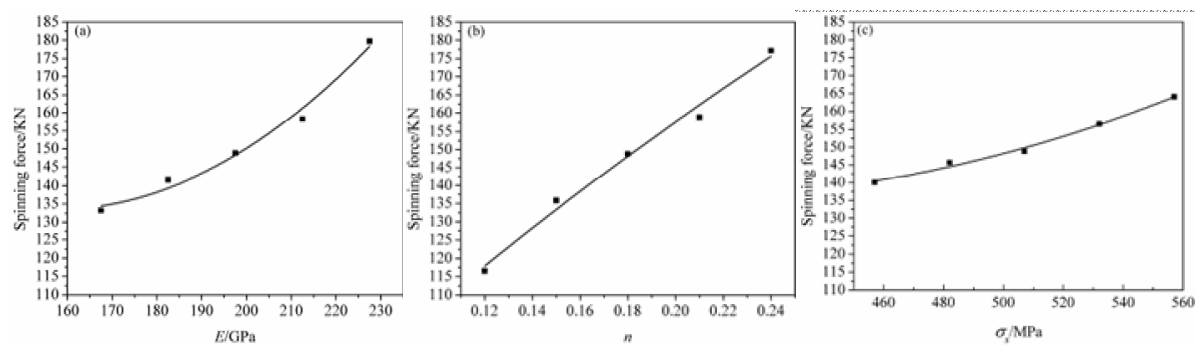

Fig.3 Influences of material parameters on spinning force: (a) $E$; (b) $n$; (c) $\sigma_{s}$ 
Fig. 4 shows that the more the $E$ is, the more the equivalent stress is, so the spinning force increases. The more the $n$ is, the more notable the effect of deformation strength is. Thus, the more the $n$ is, the more the equivalent stress and resistance of deformation is, so higher spinning force is required. The more the $\sigma_{s}$ is, the more difficult the material come into plastic deformation is. When $n$ keeps constant, the effect of deformation strength is not obvious. Thus, the more the $\sigma_{s}$ is, the more the equivalent stress is, so the more the spinning force is. In Fig.4, it can be also seen that the equivalent stress variations are more obvious than others. Hence, the influence of $n$ on the spinning force is more notable.
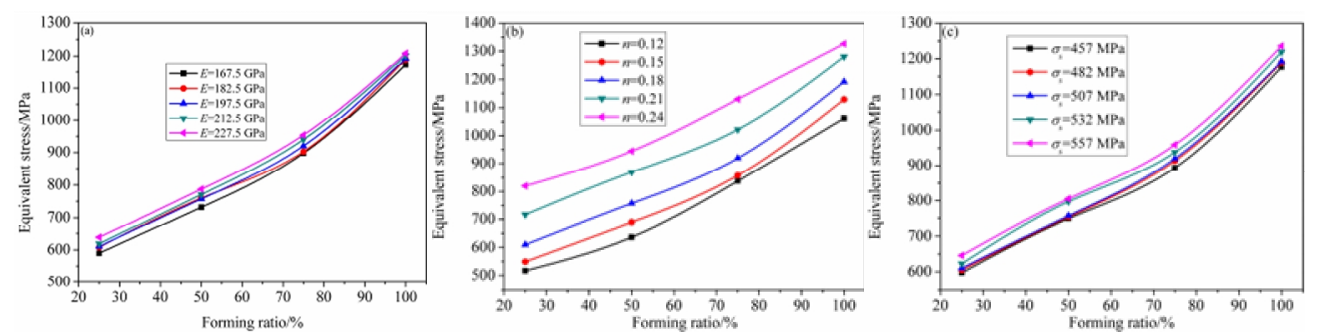

Fig.4 Influences of material parameters on equivalent stress: (a) $E$; (b) $n$; (c) $\sigma_{s}$

Degree of inhomogeneous deformation. The degree of inhomogeneous deformation $\varphi_{i d}$ is defined as the difference between the maximum and the minimum equivalent plastic strain in deformed heads, namely, $\varphi_{i d}=\varepsilon_{\max }-\varepsilon_{\min }$. During the power spinning process of LEHVT, the degree of inhomogeneous deformation $\varphi_{i d}$ is a very key parameter for selecting the materials of the blank and predicting the metal flow law of deformed heads. The lager the $\varphi_{i d}$ is, the more inhomogeneous the deformation of deformed heads is, which may cause defects in deformed heads. Fig. 5 shows that the influences of the material parameters on $\varphi_{i d}$. It can be seen that when $E$ or $\sigma_{s}$ increases but $n$ decreases, the degree of inhomogeneous deformation $\varphi_{i d}$ of deformed heads increases. And the influences of $E$ and $n$ on the $\varphi_{i d}$ are more notable than that of $\sigma_{s}$. Due to large diameter, small thickness and complicated shape of the LEHVT, there easily occurs forming defects such as wrinkling and folding, which causes higher equivalent plastic strain along the generatrix of the deformed head. Wrinkling and folding generally occur in the spinning process when the zones are subjected to the compressive hoop stress. So, the deformation of deformed heads becomes more uneven owing to the action of the compressive hoop stress. Therefore, the compressive hoop stress variations obtained from FE simulation results have been studied to evaluate the degree of inhomogeneous deformation, as shown in Fig.6.
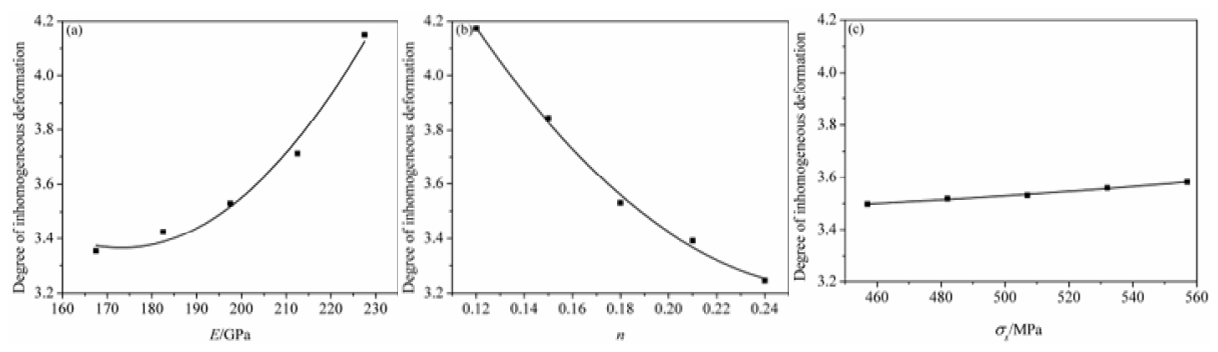

Fig.5 Influences of material parameters on degree of inhomogeneous deformation:(a) $E$; (b) $n$; (c) $\sigma_{s}$

Fig.6 (a) shows that the more the $E$ is, the more the compressive hoop stress is. Especially, at the intermediate and terminal spinning stage, the compressive hoop stress increases markedly. Fig.6 (b) shows that the less the $n$ is, the more the compressive hoop stress is. The less $n$ makes the metal flow of the exterior and the interior of deformed heads more asynchronous, which easily results in folding. Fig.6 (c) shows that the more the $\sigma_{s}$ is, the more the compressive hoop stress is. Thus, the degree of inhomogeneous deformation $\varphi_{i d}$ increases obviously. In Fig.6, it can be also seen that the compressive hoop stress variations are more obvious with changing $E$ and $n$. Hence, the influences of $E$ and $n$ on the degree of inhomogeneous deformation are more notable. 

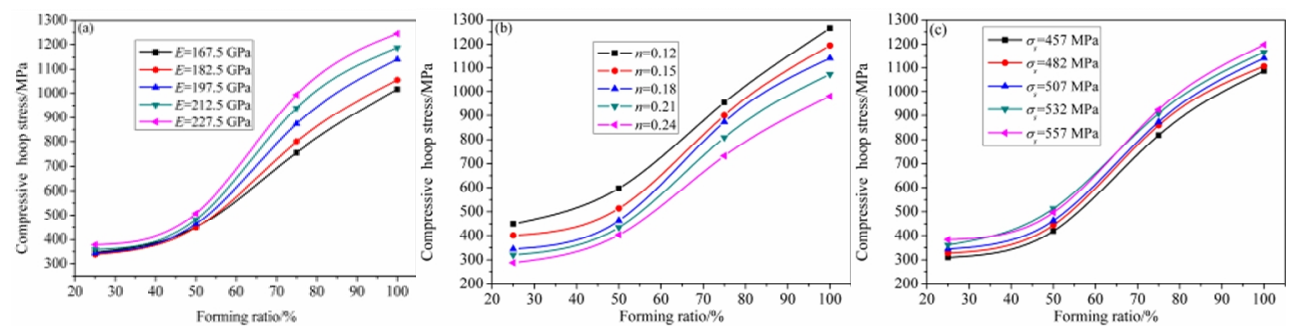

Fig.6 Influences of material parameters on hoop stress: (a) $E$; (b) $n$; (c) $\sigma_{s}$

Unfitability degree. Unfitability is an important index to judge the shape and dimensional accuracy of deformed heads. In this work, we take the unfitability degree to weigh whether the unfitability meets the demand, where the unfitability degree is presented using the maximum distance between the interior surface of deformed heads and the exterior surface of the mandrel. Fig.7 shows the influences of the material parameters on the unfitability degree. It can be seen that when $E$ decreases but $n$ or $\sigma_{s}$ increases, the unfitability degree increases. And the influences of $E$ and $n$ on the unfitability degree are more notable than that of $\sigma_{s}$. During the power spinning process of LEHVT or after unloading, considerable amounts of elastic deformation recovery may be the underlying reasons for the unfitability. Thus, the recoverable elastic strain energy distributions obtained from FE simulation results have been studied to evaluate the unfitability degree, as shown in Fig.8.
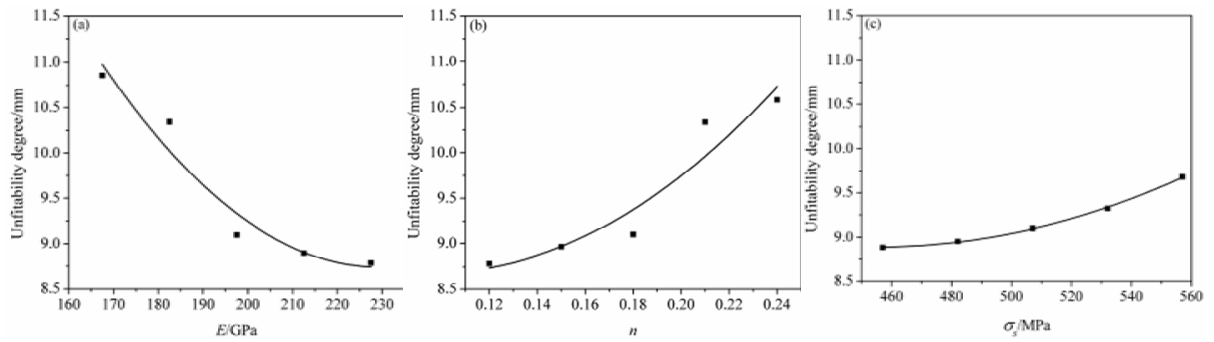

Fig.7 Influences of material parameters on unfitability degree of head: (a) $E$; (b) $n$; (c) $\sigma_{s}$

Fig.8 shows that the elastic strain energy increases wavily through the spinning process. This phenomenon may indicate that the unfitability degree of the spun head increases generally from the small end to the big end. Fig. 8 also shows that the less the $E$, the more the $n$ or the more the $\sigma_{s}$ is, the more the elastic strain energy is and the more notable the elastic recovery is, and then the unfitability degree increases obviously. As can be also observed, the elastic strain energy is sensitive to the variations of $E$ and $n$, so the influences of $E$ and $n$ on the unfitability degree are more notable.
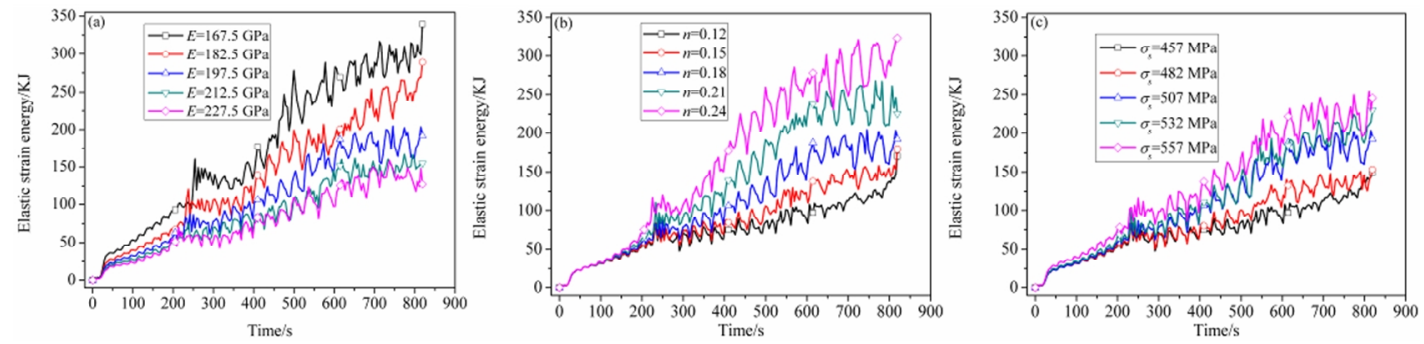

Fig.8 Elastic strain energy histories: (a) $E$; (b) $n$; (c) $\sigma_{s}$

\section{Conclusions}

1) When elastic modulus, hardening exponent or yield stress increases, higher spinning force occurs. The influence of hardening exponent on the spinning force is more notable.

2) When elastic modulus or yield stress increases but hardening exponent decreases, the degree of inhomogeneous deformation increases. The influences of elastic modulus and hardening exponent on the degree of inhomogeneous deformation are more notable. 
3) When elastic modulus decreases but hardening exponent or yield stress increases, the unfitability degree increases. The influences of elastic modulus and hardening exponent on the unfitability degree are more notable.

\section{References}

[1] J. Mackerle: Int. J. Pressure Vessels and Piping. Vol. 82 (2005), p. 571

[2] O. Music, J.M. Allwood, K. Kawai: J. Mater. Process. Tech. Vol. 210 (2010), p. 3

[3] Erik D. Szakaly, John G. Lenard: J. Mater. Process. Tech. Vol. 210 (2010), p. 868.

[4] F. Djavanroodi, M. Ebrahimi: J. Materials Science and Engineering A, Vol. 527 (2010) p. 7593.

[5] L. Huang, H. Yang, M. Zhan: J. Trans. Nonferrous Met. Soc. Vol. 18(2008), p. 674.

[6] Y.Q. Zhang, W.C. Xu, D.B. Shan: Forging \& Stamping Tech. Vol. 3 (2006), p. 74 (In Chinese).

[7] J.H. Zhang, M. Zhan, H. Yang: J. Computational Materials Science Vol. 53(2012), p. 303 